\title{
Effects of $\Delta$-9-tetrahydrocannabinol on the slope of auditory generalization gradients in rats
}

\author{
DONALD J. WEISZ and RICHARD M. VARDARIS \\ Kent State University, Kent, Ohio 44242
}

\begin{abstract}
Groups of rats were given 2,4 , or $6 \mathrm{mg} / \mathrm{kg} \Delta$-9-tetrahydrocannabinol $(\Delta-9-\mathrm{THC})$ during shuttle-avoidance training and auditory generalization testing in extinction. Although the drug had no discernible effect on acquisition performance, there was a dose-dependent influence on the slopes of the generalization gradients. It was concluded that $\Delta-9$-THC can affect the extent of auditory generalization during extinction, even when there is no apparent effect on acquisition performance. The outcomes of the study were interpreted in terms of their relevance to the drug's presumed effects on audition and various motivational processes.
\end{abstract}

Although there have been many anecdotal reports of altered auditory perception following marihuana use in humans (Tart, 1971), relatively few experimental studies have investigated possible effects of the drug on audition. In one of the early studies, Caldwell, Myers, Domino, and Merriam (1969), using standard psychophysical techniques with humans, investigated the effect of marihuana smoking on three auditory measures. No differences were obtained for absolute threshold and frequency differential threshold, but marihuana smokers had a higher differential threshold for intensity than did controls. Elsmore (1972) found no effects of $\Delta$-9-tetrahydrocannabinol $(\triangle-9-T H C$, the major psychoactive constituent of marihuana) on click frequency discrimination in two monkeys.

In a study of more complex perceptual functions, de Souza, Karniol, and Ventura (1974) investigated the effect of $\triangle-8$-THC on human tonal preferences. Subjects in 20- and 40-mg-dose groups rated high-frequency tones more pleasant than did the control subjects, but tonal preferences were similar to controls for subjects receiving 5- and 10-mg doses.

Stimulus generalization is often used to study effects of drugs on perceptual processes (Key, 1961). Recently, it has been demonstrated that stable auditory frequency generalization gradients can be obtained using a shuttle-avoidance paradigm (Rohrbaugh, Brennan, \& Riccio, 1971). The slopes of these gradients have been shown to be functionally related to various experimental manipulations (Brennan \& Riccio, 1973), suggesting that the procedure might be useful in studying drug effects on auditory processes.

To date, only one investigation of the effects of $\triangle-9-T H C$ on stimulus generalization has been

This research was supported in part by NIH Research Grant DA-00105-03 from the National Institute on Drug Abuse. Requests for reprints should be sent to Richard M. Vardaris. Department of Psychology, Kent State University, Kent. Ohio 44242. reported (Lyons, Ferraro, Lyons, Sullivan, \& Downey, 1973). Although $\triangle-9-$ THC had little effect on the slopes of floor tilt generalization gradients, there was a dose-related enhancement of peak shifts. The authors concluded that $\triangle-9-T H C$ enhanced the aversive properties of the previously nonreinforced stimulus.

The results of the above experiment suggest that cannabis may affect certain parameters of auditory function, but the nature of the change is somewhat equivocal. When the effects of $\triangle-9-T H C$ on vestibular generalization performance were investigated, the major outcome was interpreted in terms of motivational rather than sensory processes.

The purpose of the experiment reported here was to investigate the effects of $\triangle-9-T H C$ on auditory generalization in rats following acquisition of shuttle-avoidance responding to a tonal CS. Slopes of the generalization gradients were analyzed to reveal effects of the drug on frequency generalization.

\section{METHOD}

\section{Subjects}

The animals used in the experiment were 28 male albino rats from the Holtzman Company, weighing 250-310 $\mathrm{g}$ at the beginning of the study. The animals were individually housed in stainless steel cages and maintained on ad-lib Purina Lab Chow and water throughout the experiment. The animal quarters were illuminated daily from 6:00 a.m. to 8:00 p.m.

\footnotetext{
Apparatus

Training and testing were conducted in a two-compartment shuttlebox, $60.4 \times 21.0 \times 29.5 \mathrm{~cm}$. Rats could move from one compartment to the other through an opening in a center partition. The floor consisted of stainless steel rods through which a $.6-\mathrm{mA}$ scrambled constant-current shock could be delivered. Each compartment contained a photocell to detect presence of the animal. The shuttlebox was housed in a sound-attenuating chamber which was illuminated by a $15-\mathrm{W}$ bulb and ventilated by forced air.

Tonal frequencies of $1,2,4,8$, and $16 \mathrm{kHz}$ were generated by an audio oscillator and delivered through two 8-ohm speakers located $10 \mathrm{~cm}$ above the shuttlebox at each end. The average sound pressure level of the tones (measured in the shuttlebox) was $81 \pm 2 \mathrm{~dB}$ with an ambient noise level of $60 \mathrm{~dB}$.
} 


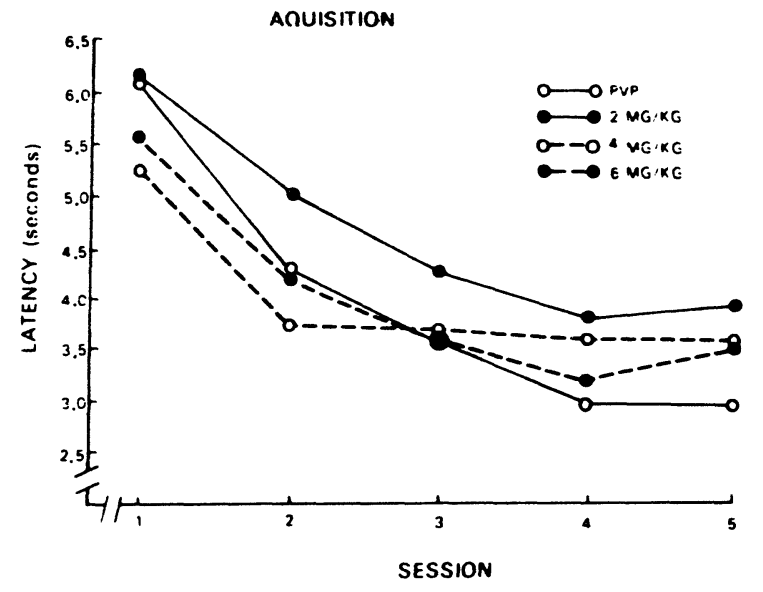

Figure 1. Mean latency of avoidance responses during acquisition as a function of days of training. The means reflect performance averaged over subjects within each of the four treatment groups.

\section{Drugs}

The trans- $\Delta$-9-tetrahydrocannabinol $(97 \%$ assay in ethanol under nitrogen) was supplied by the National Institutes of Health. Subjects received the $\triangle-9-T H C$ in the form of an aqueous suspension in the presence of polyvinylpyrrolidone (PVP) which increases the viscosity of the medium allowing $\triangle-9-T H C$ to be suspended (Fenimore \& Loy, 1971). The suspension was prepared by mixing PVP, distilled water, and ethanolic $\triangle-9-T H C$ in appropriate amounts and removing the ethanol at reduced pressure on a rotary evaporator. Working solutions were prepared by diluting the stock suspension with distilled water so as to yield three different concentrations such that the weight-proportional doses $(2,4$, and $6 \mathrm{mg} / \mathrm{kg}$ ) had an average volume of $.4 \mathrm{ml}$. Control subjects received a solution (also with an average volume of $.4 \mathrm{ml}$ ) containing $40 \mathrm{mg}$ $\mathrm{PVP} / \mathrm{ml}$. All drug solutions and suspensions were stored in the dark at $4^{\circ} \mathrm{C}$.

\section{Procedure}

Animals were randomly assigned to one of four treatment groups, such that there were seven per group. Each group received one of the following treatments: 2 . 4, or $6 \mathrm{mg} / \mathrm{kg} \Delta-9$-THC or the PVP vehicle. The drug was administered by oral intubation using a ball-tipped feeding needle $1 \mathrm{~h}$ prior to all training and testing sessions.

Subjects received 5 days of two-way shuttle avoidance trainins with 100 trials per day. The CS $(4,000 \mathrm{~Hz})$ was delivered only to the speaker above the compartment in which the subject was standing. If he failed to cross to the other compartment within $5 \mathrm{sec}$ following CS onset, an intermittent shock (UCS) was delivered to the grid bars and continued, with the CS, until the subject crossed. A crossing response during the CS-UCS interval terminated the tone and shock was not delivered. Intertrial intervals of 15,25 , and $35 \mathrm{sec}$ occurred in a random sequence.

On Day 6, a warm-up period of additional training to a criterion of five consecutive avoidances preceded generalization testing, during which the shocker was disconnected for generalization. The five tonal stimuli were presented randomly to the animals with the restriction that each block of five trials contained every frequency. Individual trials were terminated either when the subject crossed to the other compartment or when the animal failed to cross within $12 \mathrm{sec}$. Testing was terminated if a subject failed to respond with in $12 \mathrm{sec}$ to five consecutive tones in a block, or if 100 trials occurred.

Five subjects failed to meet the criterion of five consecutive avoidance responses within the first 50 warm-up trials. The responding of five animals extinguished during generalization testing before 25 test stimuli were presented. These 10 subjects were replaced by naive animals. Data for all animals that received shuttlebox training were used in the analysis of acquisition performance, whereas data from only those animals that received 25 to 100 generalization trials were included in the analysis of test performance.

\section{RESULTS}

For acquisition, mean response latencies, probabilities of conditioned avoidance responses (CAR), boluses defecated, and intertrial interval (ITI) responses were analyzed. A repeated-measures analysis of variance with unweighted means was used for each of the four analyses. Figure 1 depicts mean response latencies during acquisition training. There was a significant main effect of days $(F=70.56$, $\mathrm{df}=4 / 136, \mathrm{p}<.0001$ ) for the latency analysis. Reliable main effects of days were also found for the analyses of probabilities of CAR and ITI responses. No other effects were statistically significant in these four analyses. It was concluded that learning had occurred for all groups and that $\triangle-9-T H C$, at the doses used, had no effect on CAR acquisition, response latencies. boluses defecated, or ITI crossings during acquisition.

Figures 2 and 3 depict mean response latency for the first half and second half of testing, respectively. It may be seen in both figures that, for all groups, mean latency increased as a function of difference from the original training stimulus $(4 \mathrm{kHz})$. Differences in the slopes of the curves for the various drug doses are apparent in Figure 3, and this impression was supported by statistical analyses.

A repeated measures analysis of variance was used. The main effect (within subjects) of tones was highly significant for both first and second half data. Newman-Keuls tests revealed significant differences

FIRST HALF OF TESTING

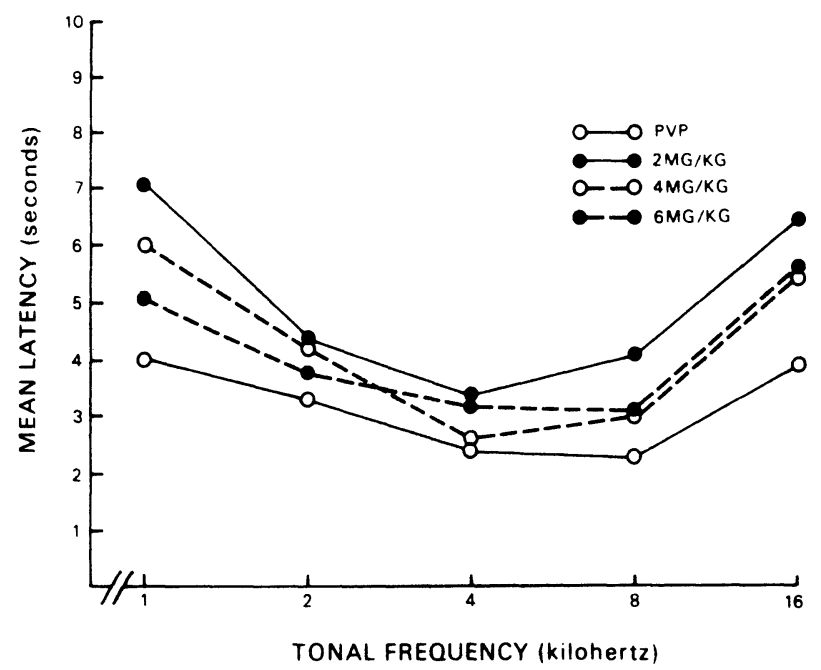

Figure 2. Mean latency of generalization responses during the first half of testing. The means reflect responses to the various tones averaged over subjects and extinction trials. The original training stimulus (CS) was $4 \mathrm{kHz}$. 
for all pairwise comparisons of mean response latency to the various tones except $4 \mathrm{kHz}$ vs. $8 \mathrm{kHz}$, and $1 \mathrm{kHz}$ vs. $16 \mathrm{kHz}$ (the endpoints of the gradients) for both first and second half data. The between-subjects main effect of drug and the interaction of Drug by Tone were also significant for the second half data $(\mathrm{F}=4.338, \mathrm{df}=3 / 24, \mathrm{p}<.05)$, for the main effect of drug and $(\mathrm{F}=2.905, \mathrm{df}=12 / 96, \mathrm{p}<.01)$ for the interaction. Newman-Keuls comparisons were carried out for the simple effects of drug at each tone for the second half data. There were significant differences between the PVP control and the various $\triangle-9-T H C$ groups for the endpoints of all the gradients, except for the $6 \mathrm{mg} / \mathrm{kg} \Delta-9-\mathrm{THC}$ dose at $1 \mathrm{kHz}$. Also there was a significant difference between the $2 \mathrm{mg} / \mathrm{kg}$ $\triangle$-9-THC and $6 \mathrm{mg} / \mathrm{kg} \triangle-9-\mathrm{THC}$ doses at $1 \mathrm{kHz}$. However, there were not differences in response to the original training stimulus. From these analyses, it was concluded that reliable generalization gradients had been obtained, and that in the second half, $\triangle-9-T H C$ had a steepening effect on them, such that the 2 - and $4-\mathrm{mg} / \mathrm{kg}$ doses produced the steepest gradients. All three drug doses produced steeper gradients than the vehicle control.

A repeated measures analysis of variance and Newman-Keuls tests were performed on the mean latencies for the combined halves of testing. The results were identical with those of the second half of testing. No differences were found during testing between drug groups on ITI responses and boluses defecated.

\section{DISCUSSION}

The results of this experiment demonstrated that $\triangle$-9-THC can affect the slope of generalization gradients in a dose-dependent manner. However, the nature of the dose-dependency is unusual in the sense that the steepest gradients occurred for the intermediate doses. This relationship could be explained by the hypothesis that steepness is a nonmonotonic or inverted- $U$ function of dose. It is possible that the present study used doses associated with the descending limb of this function. Although it is relatively rare for oral doses of $\triangle-9$-THC less than $2 \mathrm{mg} / \mathrm{kg}$ to show consistent psychological or biological activity, the possibility exists that the auditory perceptual system is particularly sensitive to the drug, such that generalization gradients might be steepened by doses smaller than those used in the present investigation.

This effect of $\triangle$-9-THC on audition presents a difficult problem of interpretation. It may be that at moderate doses (i.e., $2 \mathrm{mg} / \mathrm{kg}$, oral) the drug optimally "sharpens" the tuning of the auditory system such that perceived contrast among neighboring frequencies in the spectrum is increased. Such a process might have the effect of enhancing the detection of specific sounds embedded in a complex auditory input.

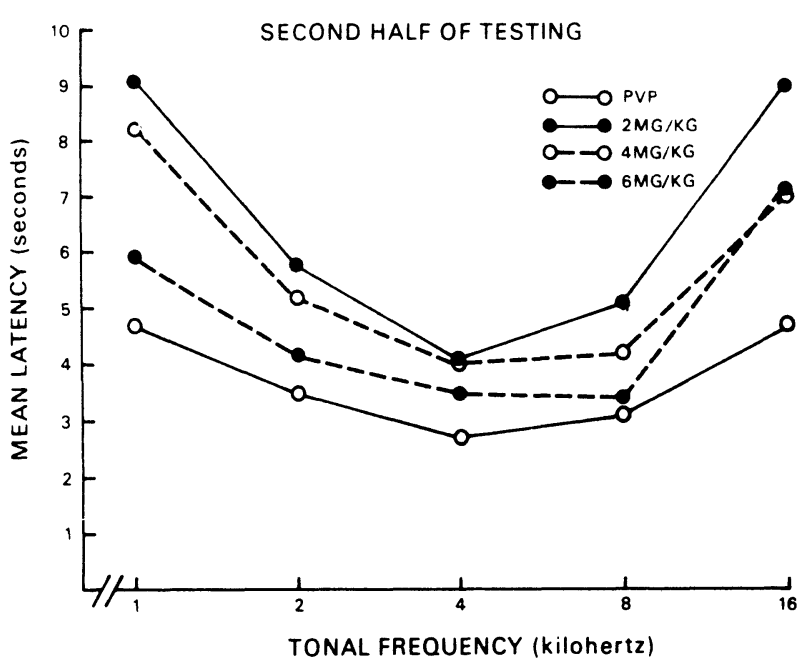

Figure 3. Mean latency of generalization responses during the second half of testing. Mean responses to the tones are averaged for extinction trials and subjects within groups. The CS during training was $4 \mathrm{kHz}$.

However, cannabis is thought to affect many other psychological processes that could result in altered slopes of generalization gradients, such as resistance to extinction (Jaffe \& Baum, 1971) or pharmacological factors such as analgesia (Bicher \& Mechoulam, 1968). Although there were no apparent drug effects on resistance to extinction in the present experiment, it is possible that the arbitrary cutoff at 100 extinction trials masked drug effects that could have occurred with more trials. There is a tendency for generalization gradients to steepen with extinction and if extinction were accelerated by $\triangle-9-T H C$, the gradients for the drug group might be steeper than for the control group.

The acquisition data suggest that the results of the present experiment cannot be explained in terms of the drug's analgesic activity. There were no reliable differences between the drug and control groups in acquisition performance or in responding to the original stimulus (CS) during generalization testing. Additional support for the interpretation that $\triangle$-9-THC did not reduce the aversiveness of the UCS comes from the observation that there were no reliable differences between the drug groups and the control group in fecal bolus counts or ITI crossings at any time in the experiment. These measures may be regarded as indices of possible emotionality produced by the UCS and the conditioning procedure. Therefore it seems unlikely that the drug affected motivational levels or efficacy of reinforcement in the present study.

In view of the pattern of results obtained, it seems probable that $\triangle-9-$ THC affected the auditory system in some way, although certain alternative explanations may not be ruled out without further research. In addition, these findings confirm the studies of Rohrbaugh et al. (1971) and Brennan and Riccio (1973); stable auditory generalization gradients 
may be obtained with instrumental avoidance procedures.

\section{REFERENCES}

Bicher, H. I., \& Mechoulam, R. Pharmacological effects of two active constituents of marihuana. Archives Internationales de Pharmacodynamie et de Therapie, 1968, 172, 24-31.

Brennan, J. F.. \& Riccio, D. C. Stimulus control of avoidance in rats following differential or nondifferential Pavlovian training along dimensions of the conditioned stimulus. Journal of Comparative and Physiological Psychology, 1973, 85, 313-323.

Caldwell, D. F., Myers, S. A., Domino, E. F., \& Merriam, P. E. Auditory and visual threshold effects of marihuana in man. Perceptual and Motor Skills, 1969, 29. $755-759$.

de Souza, M. R. C., Karniol, I. G., \& Ventura, D. G. Human tonal preferences as a function of frequency under delta-8-tetrahydrocannabinol. Pharmacology, Biochemistry and Behavior, 1974, 2, 607-611.

ELSMORE. T. F. Effects of delta-9-tetrahydrocannabinol on temporal and auditory discrimination performance of monkeys. Psychopharmacologia, 1972, 26, 62-72.

FEnimore, D. C., \& LOY, P. R. Injectible dispersion of
$\Delta \overline{9}$-tetrahydrocannabinol in saline using polyvinylpyrrolidone. Journal of Pharmacy and Pharmacology, 1971, 23, 310.

HENRIKSSON, B. G., \& JÄRBE, T. The effect of two tetrahydrocannabinols $\left(\triangle^{9}-\right.$ THC and $\Delta^{8}$-THC) on conditioned avoidance learning in rats and its transfer to normal state conditions. Psychopharmacologia. 1971, 22, 23-30.

JAFFE, P. G., \& BAUM, M. Increased resistance-toextinction of an avoidance response in rats following the administration of hashish resin. Psychopharmacologia, 1971, 20, 97-102.

KEY, B. J. The effect of drugs on discrimination and sensory generalization of auditory stimuli in cats. Psychopharmacologia, 1961, 2. 352-363.

Lyons, J., Ferraro, D. P., Lyons, J. E., Sullivan, J. G. \& DOWNEY, D. Effects of delta-9-tetrahydrocannabinol on stimulus control. Bulletin of the Psychonomic Society, 1973, 2, 302-304.

Rohrbaugh, M., Brennan, J. F., \& Riccio, D. C. Control of two-way shuttle avoidance in rats by auditory frequency and intensity. Journal of Comparative and Physiological Psychology, 1971, 75, 324-330.

TART, C. T. On being stoned. A psychological study of marihuana intoxication. Palo Alto, California: Science and Behavior Books. 1971.

(Received for publication September 15, 1975; accepted September 15, 1975.) 